\title{
An Enterprise Security Program and Architecture to Support Business Drivers Brian Ritchot
}

\author{
"We will bankrupt ourselves in the vain search for") \\ absolute security.
}

Dwight D. Eisenhower (1890-1969)

34th President of the United States

\begin{abstract}
This article presents a business-focused approach to developing and delivering enterprise security architecture that is focused on enabling business objectives while providing a sensible and balanced approach to risk management. A balanced approach to enterprise security architecture can create the important linkages between the goals and objectives of a business, and it provides appropriate measures to protect the most critical assets within an organization while accepting risk where appropriate. Through a discussion of information assurance, this article makes a case for leveraging enterprise security architectures to meet an organizations' need for information assurance. The approach is derived from the Sherwood Applied Business Security Architecture (SABSA) methodology, as put into practice by Seccuris Inc., an information assurance integrator. An understanding of Seccuris' approach will illustrate the importance of aligning security activities with high-level business objectives while creating increased awareness of the duality of risk. This businessdriven approach to enterprise security architecture can help organizations change the perception of IT security, positioning it as a tool to enable and assure business success, rather than be perceived as an obstacle to be avoided.
\end{abstract}

\section{Introduction}

Many organizations find that their existing security controls are preventing them from getting something done or are reducing their effectiveness. Conversely, an organization may question if there is sufficient protection for information that is to be shared with a new business partner, customer, or the general public. If a critical system is compromised, what will be the business impact?

In order for a security program to be effective, it must demonstrate value to the business while avoiding the traditional pitfalls associated with the perception of security being an inconvenience and an obstacle to effective business operations. Security practitioners are challenged to consider security in the context of the business and understand the duality of risk: some risks represent business opportunities and should therefore be accepted. However, risk avoidance is a common practice within IT organizations, where security expenditures, policies, procedures, and technologies are not proportional to the risk appetite of the business. When security controls become overly intrusive to employees of a business, and in fact impede business operations, individuals will seek the means to bypass these controls. This desire to avoid security is due to the perception that security is an obstacle. As a result of this security avoidance, new risks are introduced, however are not known to the security team and cannot be monitored and managed.

The situation describes a common struggle faced by most organizations. Organizations strive to achieve the appropriate balance between security controls to protect business information, while also allowing their employees to be productive and share information easily. Achieving this balance requires information assurance. 


\section{An Enterprise Security Program and Architecture to Support Business Drivers}

\section{Brian Ritchot}

This article will provide an initial understanding of information assurance and present the case for leveraging enterprise security architectures to meet an organization's need for information assurance. The approach is derived from the Sherwood Applied Business Security Architecture (SABSA; tinyurl.com/9hg3se2) methodology, as put into practice by Seccuris Inc. Seccuris (seccuris.com) is a Canadian information assurance integrator that helps organizations achieve their business goals through effective management of information risk. To help customers effectively manage risk and capitalize on business opportunities, Seccuris has come to rely on business-driven enterprise security architectures. Seccuris adopted the SABSA methodology for enterprise security architectures to provide organizations with the often-missing critical link in effective cyberthreat mitigation. This missing link is an appropriate understanding of business goals and a structured repeatable process with which to identify assets of critical value to the organization and deliver appropriate safeguards within an established risk appetite.

An understanding of Seccuris' approach will illustrate the importance of aligning security activities with highlevel business objectives while creating increased awareness of the duality of risk. The business-driven approach to enterprise security architecture can help organizations change the perception of IT security, positioning it as a tool to enable and assure business success, rather than an obstacle to be avoided.

The article is intended for senior executives within an organization who are trying to rationalize an appropriate balance between the protection and availability of information that supports the business. The article will also help security practitioners, in particular security architects, understand how to align security initiatives with business goals to deliver an effective security program.

\section{Information Assurance}

Information assurance relates to the management of risk and security related to the use, processing, storage, and transmission of data. It is part of a broader category, known as information security, that is predominantly focused on IT security controls and processes. IT security deals primarily with the confidentiality, integrity, and availability of information and provides mechanisms to protect these aspects. When information is compromised, the result is a change in state of one of these aspects.
1. Confidentiality: ensures that privileged or sensitive information is accessible only to those individuals with a valid requirement to view and access the information. It is particularly important when concerning personal information, intellectual property, and classified or sensitive information in a government context.

2. Integrity: refers to a lack of corruption in data or overall consistency. When integrity of information is compromised, it creates a lack of trust wherein data may have been manipulated, changed, or deleted.

3. Availability: relates to having access to authorized information when it is required. Should information be affected so it cannot be accessed when needed and authorized, then availability has been compromised.

Information risk arises when the confidentiality, availability, or integrity of data can be compromised. To mitigate risk, controls can be developed and implemented to provide increased assurance of information. A control is a safeguard or countermeasure designed to avoid, minimize, or counteract risk.

The practice of information assurance relies on the identification of risk and the application of appropriate controls. However, over time, this practice has come to be categorized as "risk adverse" and to be seen as an impediment to business effectiveness. Information security professionals are labelled as obstacles to successful implementation and delivery of IT solutions. The resulting business culture is reluctant to involve and solicit input from IT security teams, because the input can create business risk for a project and delay implementation. This view comes from the practice of creating a risk-avoidance approach to information security, based solely on technical threats, identification of risk, and use of as many controls as possible to mitigate risk. The end result of this practice is a security program that fails in its effectiveness, given reluctance at an organizational level to involve security in the early stages of projects and planning. True information-assurance practices must also recognize the value and importance of making information available and establishing safe information-sharing practices.

In the first 6 months of 2010, McAfee (2010; tinyurl.com/ n7rykk6) discovered over 10 million new pieces of malware. According to the US Intellectual Property Commission Report (IP Commission, 2013; tinyurl.com/ pnyjnod), hundreds of billions of dollars are lost each 


\section{An Enterprise Security Program and Architecture to Support Business Drivers}

\section{Brian Ritchot}

year to the theft of intellectual property. Increasingly, this theft is the result of cyberattacks against United States' electronic infrastructure. Sophisticated samples of malware have been discovered in recent years, with demonstrated capability to attack SCADA control networks (tinyurl.com/jcrlz) and negatively impact critical infrastructure. The two most notable examples are "Stuxnet" and "Flame" (Klochender, 2013; tinyurl.com/ 16vb6ja). Together, all of these examples illustrate a failing in existing information assurance practices and a rise in the sophistication and capabilities of cyberadversaries. When faced with these challenges, many organizations may default to implementing more security controls to minimize vulnerabilities and deny cyberadversaries access to systems. An approach focused solely on controls will ultimately prove unsuccessful given the resourcefulness and capabilities of malicious threat actors, as demonstrated in the examples above. Through a history of assisting customers across multiple sectors and varying levels of government, Seccuris has identified a common thread: while most organizations are security conscious and acknowledge the need for good IT security practices, they lack the necessary knowledge needed to build effective security programs.

Security programs should be designed to provide appropriate protection for information and information assets. This protection should be tailored to the environment, which requires the identification of what information is most vital to an organization. Without a clear understanding of priorities for information, and information security, organizations are incapable of prioritizing control improvements. A lack of a structured security architecture impacts all aspects of an organization's IT security program, including threat monitoring, vulnerability management, identity management, and incident response just to name a few. Effective security operations require effective security architecture.

\section{Sherwood Applied Business Security Architecture (SABSA)}

When security is found to be cumbersome or intrusive into business practices, loopholes and shortcuts are taken to bypass the implemented controls, creating increased risk that is not accounted for and is easily capitalized upon by threats seeking to compromise the confidentiality, integrity, and availability of data. The Sherwood Applied Business Security Architecture (SABSA) methodology for an enterprise security architecture and program can be leveraged to address this shortcoming (Sherwood, et al., 2009; tinyurl.com/mkggknj).
In essence, the SABSA approach is centered on making security a business enabler rather than an obstacle and avoidable inconvenience. The SABSA approach creates an understanding of an organization's business objectives and provides a structured approach to designing a security program that supports these objectives. Security does not hinder business objectives, but instead provides assurances around operational risk that could negatively impact the business and, in fact, enables the organization to take on new strategic opportunities.

SABSA is a unique approach to information assurance because it seeks to align security programs with an organization's fundamental business objectives and drivers. In doing so, the SABSA approach treats risk as something that can not only hinder a business, but can also enable new opportunities. It is necessary for organizations to accept risk in order to do business and be effective. Embracing the right type of risk has the potential of leading to good fortune for a business (Card, 2013; timreview.ca/article/696) .

An important element to consider when selecting an architectural framework for security, is that many organizations already have an established IT architecture program to facilitate delivery of IT projects. Some existing architectural frameworks include the Open Group Architecture Framework (TOGAF; tinyurl.com/yj72xcf) and the Zachman Framework (tinyurl.com/4axvn2e); however, such frameworks do not traditionally address security requirements. Furthermore, many organizations implement service-management programs to manage and operate IT systems and services. The Information Technology Infrastructure Library (ITIL; tinyurl.com/ mukhg) is an example of such a service-management framework. SABSA is unique among architectural frameworks in that it does not seek to replace or interfere with these existing frameworks and practices, but instead integrates with them and provides the necessary tools to align and support existing architectural programs. This ease of alignment with existing frameworks and the focus on leveraging security as a business enabler are key criteria that affected Seccuris' decision to leverage SABSA as a framework for delivering enterprise security architectures.

To make security relevant to all stakeholders within an organization, the SABSA framework introduces a layered approach to architecture. Each layer corresponds to a different player's view within the organization as it relates to specifying, designing, constructing, and operating a security architecture, as shown in Figure 1. 


\title{
An Enterprise Security Program and Architecture to Support Business Drivers
}

\section{Brian Ritchot}

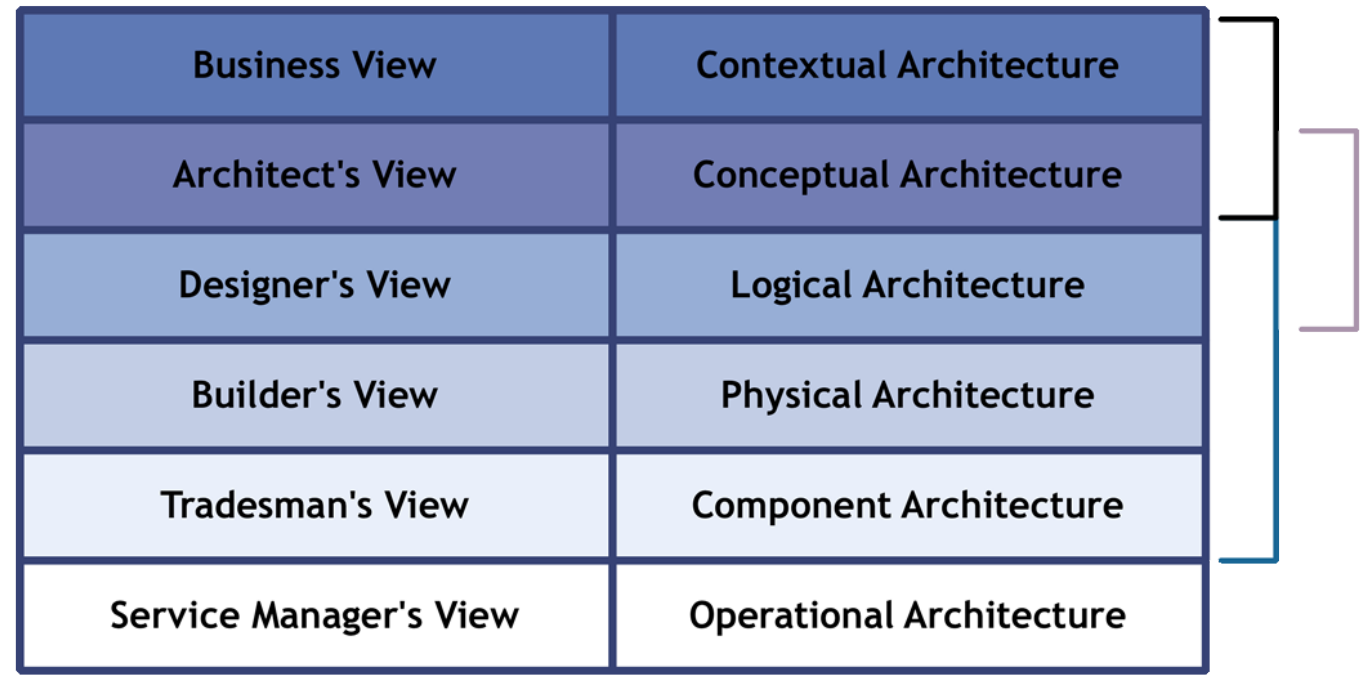

\author{
Enterprise \\ Security Program \\ \& Architecture \\ Security Services \\ Architecture \\ (Service) \\ Security Solutions \\ Architecture \\ (Solution)
}

Figure 1. The SABSA model for security architecture

For each of the architectural views in Figure 1, SABSA encourages security architecture to consider the following key questions:

1. What are you trying to protect at each layer? (Assets)

2. Why are you protecting these assets? (Motivation)

3. How will you achieve your objective? (Process)

4. Who is involved in applying security? (People)

5. Where are you applying security? (Location)

6. When are you applying security?(Time)

SABSA provides the theory and background knowledge essential to delivering business-driven security architectures; however. the practice of delivering the architecture is left up to the security practitioner. As described in the next section, Seccuris has developed a repeatable process and procedures that answers the above questions for each layer of the architectural model.

\section{Enterprise Security Architecture: Establishing the Business Context}

A business-driven approach to enterprise security architecture means that security is about enabling the objective of an organization by controlling operational risk. This business-driven approach becomes a key differentiator to existing security practices that are focused solely on identifying threats to an enterprise and technical vulnerabilities in IT infrastructure, and subsequently implementing controls to mitigate the risks introduced. A purely threat-based approach to risk management fails to enable effective security and business operations. The term security will carry very different meanings to different organizations. For example, consider security as it relates to a military organization and security related to an online retailer that processes credit card information. The business models for these two organizations will be very different and, as a result, the security programs should be unique and relevant to their underlying businesses. A military organization may determine that the most critical asset to protect is the life of its soldiers as they are engaged in military operations. To provide assurance as to the safety of a soldier, complex security architectures are needed to protect information and information systems that could impact the soldiers' safety. Solutions could range from ensuring that logistic systems that manage the delivery of supplies, food, and ammunitions remain available and that data integrity is protected to protecting confidentiality of mission plans and military intelligence that, if compromised, could cause considerable harm to war fighters. Conversely, an online retailer is likely most concerned with compliance with standards set by the payment card industry. These standards are tailored to protect the confidentiality of personal information and the integrity of transactions. An online retailer may have lower thresholds for availability then a military logistics system. The needs for confidentiality, availability, and integrity of data must be balanced and appropriate to the business activity. 


\section{An Enterprise Security Program and Architecture to Support Business Drivers}

\section{Brian Ritchot}

Developing a security architecture begins with an understanding of the business, which is achieved by defining business drivers and attributes. A business driver is related to the organization's strategies, operational plans, and key elements considered critical to success. A business attribute is a key property of the strategic objectives that needs to be enabled or protected by the enterprise security program. An organization's senior executives, who set the long-term strategy and direction of the business, can typically provide knowledge regarding business drivers. The drivers are often reflected in an organization's mission and vision statement. Consider our military organization, which may have a strategic objective of "operational excellence". This business driver can be distilled into relevant attributes that require assurance to satisfy the overarching business driver. Conversely, the online retailer may have a strategic objective of being "customer focused", as expressed in their vision statement to provide a superior online shopping experience.

Business attributes can generally be identified through an understanding of the business drivers that are set by the top levels of an organization. Security architects will often conduct structured interviews with senior management in order to identify business attributes by determining the essence of what is conveyed by high-level business drivers. In the example of the business driver labelled "operational excellence", the executives might be referring to the availability, reliability, and safety of their operations and resources. In this case, the business attributes defined are "available", "safe", and "reliable". Each attribute is then linked to the business driver they support. This pairing of a business driver and attribute results in the creation of a proxy asset. Again, building on our example, a sample proxy asset is "operational excellence" with the attribute of "available". Each proxy asset is owned by the organization and is assessed as having value to them. The fact that the proxy asset has value sets the requirement that it should be protected. The value of these proxy assets is difficult to define given that they are often intangible and exist at a very high level. Despite being unable to assign a monetary value to a proxy asset, it is still possible to identify risks that may act against the asset. Our online retailer may have attributes of "confidential", "reputable", and "error-free".

An inventory of proxy assets can be maintained by the security architect and will be considered as key assets to the organization. This is later used to conduct a business threat and risk assessment to identify risks to the business. It is through a business threat and risk assess- ment that the sometimes-competing aspects of confidentiality, integrity, and availability can be reconciled. When the overall objective and needs of a business are understood, through proxy assets, then impact can be understood as it relates to confidentiality, integrity, and availability. Understanding of the business helps prioritize which of these elements is most important, and which aspects of the business are most in need of protection.

\section{Identification of Risk}

Traditional threat-based risks are those that can be mitigated via a control because they would result in the loss of value. Many organizations rely on threat risk assessment to create an inventory of threats that may have a negative impact on the business. These threats are then mapped to vulnerabilities that, when exploited, result in a compromise to the organization's business. Managing this risk often relies on the deployment of security controls that offer a form of mitigation. Consider, for example, an external website that has a technical vulnerability that could result in a threat that exploits this vulnerability, such as a denial-of-service attack, which would render the website inaccessible. Threat-based risk analysis would identify the threat and provide recommendations on control improvements to decrease the risk. This may include the deployment of a web-application firewall to provide intrusion-prevention capabilities, increased network monitoring, the deployment of additional firewalls, and software upgrades to reduce the vulnerability.

The traditional approach described above successfully identifies risk based on an analysis of possible threats and provides the means of mitigating that risk. The relation of the risk to business impact, however, is missing in this approach. Perhaps the server is used for testing with a small number of business partners, and denial of service is not a risk of importance. The implementation of controls sometimes does not offer protection for what is truly important to the business. Controls can increase complexity within the network and incur costs that could have been avoided. When identifying risk using only threat-based approaches, key information is likely missing, which could have informed a security architect on where to prioritize control improvements. Another problem with threat-based approaches is that they do not consider the potential opportunity that can be realized when embracing risk.

Consider the non-traditional idea that risks can also be categorized as opportunity-based - this perspective is 


\section{An Enterprise Security Program and Architecture to Support Business Drivers}

\section{Brian Ritchot}

lacking from traditional information assurance practices. An opportunity-based risk can increase the value of an asset. This approach enables us to understand the duality of risk. Some risks should be mitigated, while others might be accepted as something that cannot necessarily be avoided; businesses always operate with some level of inherent risk.

When a business and security architects understand the duality of risk, they can also focus on risk acceptance rather than just risk avoidance. To this end, the organization develops key performance indicators (KPIs) and key risk indicators (KRIs). KPIs are measures of the value and performance of business attributes in the context of the business driver. KRIs are measures of risk, and they establish risk thresholds to provide early warning when a risk will exceed an organization's risk tolerance.

In our military example, we identified "operational excellence" as a business driver and "available" as an attribute. Developing a KPI around the availability of operational systems would allow the organization to measure the availability and uptime of a key business application. The performance of this application could be tracked over time to ensure that it remained available and supported the business driver of "operational excellence". Conversely, the same example could be considered from a different angle and result in the development of a KRI. A KRI differs from a KPI in that it establishes a threshold or condition that creates a warning state. Monitoring of KRIs provides an indication when risk is about to exceed established tolerance levels. In our example, a KRI might enable ongoing measurement of the time that an application is inaccessible. The system may be inaccessible due to technical circumstances such as network failure, software updates, or other IT incidents. Measuring the time that an application is inaccessible will facilitate identification of an established threshold that sets unacceptable behaviour. When the key business application approaches this threshold, alerts can be generated to warn that the established risk tolerance is soon to be exceeded.

In our online retailer example, the attribute "error free" can apply to the processing of financial transactions. In order to provide a consistent experience for users and to maintain customer confidence, the online retailer wants to ensure that any transactions are without error. A KRI can be created to capture any time a financial transaction is disputed due to a potential error. It is likely that a very low threshold would be established and any errors would trigger appropriate response to investigate and remediate the cause of the error.

Regardless of the indicator selected (i.e., KPIs or KRIs), when considered in the context of business drivers and attributes, it becomes possible for security to have sufficient information to consider risk within the context of business objectives. This understanding of risk will contribute to an overarching model of business risk, which is needed for a security architect to successfully develop security architecture at an enterprise level.

\section{Creating a Model of Business Risk}

A model of business risk provides a mechanism for quantifying risk and ensuring that it remains relevant to business drivers and attributes (as described above). The business-risk model builds on the understanding of risk, centered on the established proxy assets as well as KRIs and KPIs. In addition to the proxy assets and KPIs and KRIs, there are other models that also must be developed and understood to complete the businessrisk model: i) trust models and business relationships, ii) threats operating against the business, and iii) safeguards that have been implemented. This approach is similar to a threat risk assessment; however, the difference is that a threat risk assessment measures the risk to a system or IT environment. An enterprise security architecture measures the risk to the proxy assets that represent the organization's business.

\section{Trust models}

Trust needs to be considered in the context of the overall business as a business attribute, not a technical one. Whenever two or more entities are required to interact and exchange information, trust must first be established between the two entities. Trust can be established through registration of the entity by the other. The registering entity will then trust the entity that has been registered, based on assurance mechanisms. The levels of assurance required to establish this trust vary, based on the degree of risk involved.

As an example, consider a shopkeeper selling lottery tickets to a customer. For the shopkeeper to trust the customer, they require a valid form of identification, such as a driver's licence, to validate the customer's age. The decision to require the driver's licence is based on a risk decision taken by the shopkeeper on whether the individual appears of age or not. Conversely, the customer must trust the shopkeeper as a valid merchant authorized to sell lottery tickets. This trust is established through the clear display of the lottery licence 


\section{An Enterprise Security Program and Architecture to Support Business Drivers}

\section{Brian Ritchot}

issued and validated by the government. Once these assurances are validated, the shopkeeper and customer establish a two-way trust relationship to complete the transaction.

Understanding business risk requires that relationships internal and external to a business are properly understood. Evaluation is required to gather information related to the criticality of a relationship, the sensitivity of information shared, and existing methods of assuring trust between the parties involved in a relationship. A security architect can explore the relationships both internal and external to an organization to identify the points where information is exchanged. This information can be used by a security architect to layer logical relationships to the physical IT environment to prioritize the placement of controls to assure trust and protect information as it is exchanged as part of business relationships.

\section{Threat models}

Threats are entities or things, operating against a business, that cause damage or harm to an organization. Developing an understanding of threats is an important step in developing awareness of business risk. Typically, safeguards are implemented to mitigate damage caused by a threat that is exploiting a vulnerability. A threat may be a deliberate action taken by an entity or it may be accidental, based on an unintentional realization of a scenario that creates risk. Natural hazards, such as fire and flood, are threats that may impact a business. Understanding these threats, the likelihood of them operating against a business, and the gravity of consequences should they succeed, helps in creating a prioritization of threats based on the impact and likelihood of being realized.

In the case of our military organization a threat to availability may materialize from a hostile government agency or military that would seek to disrupt the availability of key systems essential to effective military operations. The online retailer would not necessarily be concerned with military threats, and would instead consider organized crime as a threat that would seek to abuse the technical application in the retailer environment for the purposes of financial gain. This threat may include gaining access to confidential customer data for identity theft, or exploiting application vulnerabilities to order receive goods without paying full price. The threat model can be made relevant to the organization by considering threats that affect that proxy assets defined earlier in the process.

\section{Safeguards}

The final model required to establish the business risk model is an understanding of existing safeguards and any gaps that exist in mitigating risk associated with threat activity. Industries follow various standards of best practice and frameworks to assess their relative maturity regarding security controls and safeguards implemented to mitigate risk.

A popular general purpose control framework is ISO 27002 (tinyurl.com/lcz75nz), which is provided by the International Organization for Standardization (ISO). This standard provides industry best practices for information security management. Other control frameworks specific to industries include the North American Electrical Reliability Corporation (NERC) Critical Infrastructure Protection (CIP; tinyurl.com/l22ggon) standard and the International Society of Automation (ISA; isa.org) standards. Selecting the appropriate framework is an important step in conducting a review and identifying relevant gaps. Each business will have a unique set of controls relevant to their industry and the regulated protection required. Once a control framework is selected, a review of the controls can be undertaken along with assessment of the maturity level for each control and the extent to which it is implemented in the business.

Once all data around risks, relationships and trust, threats, and control effectiveness have been gathered, the inherent risk to a business can be described and represented. An initial assessment is undertaken where the business impact of a risk being realized is considered, irrespective of any existing controls, to quantify the severity of risk. Based on this understanding of the inherent risk, a security architect can identify areas for improvement that will manage risk and establish acceptable thresholds. Inherent risk is a measure of the risk to the enterprise prior to any controls being implemented.

Risk can be classified on a scale tailored to meet an organization's need; however, fundamentally, risk will be quantified as negligible, acceptable, significant, or severe. In the case of negligible risk, no action is required, whereas acceptable risk requires monitoring to ensure it remains at an acceptable level. Significant and severe risks require action to establish an appropriate risk threshold in which the business is comfortable operating, to maximize opportunities. The resulting risk score is known as residual risk, which is the risk that remains after security controls and improvements are selected, approved, and implemented in an environment. 


\section{An Enterprise Security Program and Architecture to Support Business Drivers}

\section{Brian Ritchot}

Improvements to controls can be simulated and new risk scores can be calculated to develop risk-reduction strategies. This process shows how security improvements can affect enterprise-wide risk. Because various improvements carry different complexities and cost, multiple models can be constructed to show options and potential benefits over time as control improvements are made. This approach allows an understanding of risk appetite and provides the overall business-risk model.

\section{Using a Business-Risk Model to Drive Security Architecture}

Once a business risk model has been completed, a security architect can leverage this information to create logical security services. A logical service is specified independent of any physical mechanism that might be used to deliver the service. Most importantly, a logical service is driven from the business attributes and the business-risk model. A security service is therefore a combination of security controls that work together to support the delivery of a valued business service. A number of security services can be developed to provide "defence in depth" and increase the overall assurance of information that is important to the business. Sample security services that can be created may include threat management, vulnerability management, and network access. These services will form part of a security-services catalogue and, as a result, services can be selected and implemented based on the needs of a specific business initiative. Unlike traditional security controls, these services are derived from business drivers and attributes, and they provide traceability to the business objectives.

The services to be designed will draw on the information gathered in the business-risk model, particularly the understanding of relationships expressed as physical boundaries where information is shared, threats that are operating against the environment, and opportunities for control enhancements to mitigate the risks introduced by threats. The added benefit of developing security services based on the business-risk model is that full traceability can be maintained, thereby demonstrating that security initiatives are tied to supporting an organization's business and effectively managing both threat- and opportunity-based risk.

\section{Relevance to Cybersecurity}

Although the concept of enterprise security architecture does not provide a concrete technical tool with which to counter advanced persistent threats or zeroday attacks, it provides a critical tool for identifying and assessing assets of value to an organization. This step is often missing when developing security programs, which can lead to the deployment of controls without a proper understanding of how they can hinder or support the overarching business objectives.

Consider our two examples: the military organization and the online retailer. Both are likely to have an Internet-accessible website. The purpose of these websites would be very different in both cases. For the military, it likely provides general information at the unclassified level to the general public; it would not likely be used for operational missions and would be in a separate network segment from critical operational systems. The online retailer, however, would use the website as a front-end to their e-business, where the website provides customers with the ability to browse and purchase merchandise. If both organizations have an enterprise security architecture, they are better equipped to respond to, and deal with, threat activity when it materializes against the website.

In the event of a zero-day attack against their webserver, the military would realize that the attack did not affect any critical information and was not related to a key relationship that was foundational to success of the organization. During incident response, the effort and tools used to respond would be appropriate, and control improvement would be balanced based on cost, impact, and risk tolerance. Although the reputation of the organization may be damaged should the information become public, the overall business impact would be minimal. The online retailer, however, would need a much different response based on their business needs. Furthermore, the logical security services that we alluded to would be geared towards protecting confidentiality and ensuring error-free processing. Although the discussion of logical-service design falls outside of the scope of this article, consider briefly the concept of vulnerability management. The online retailer would likely perform regular web-application assessments and vulnerability testing against its website and web application to appropriately protect customer data. The military organization, also implementing a vulnerability-management program, would most likely scan the website on a much less frequent basis and focus efforts on securing mission-critical systems, requiring high availability.

An enterprise security architecture helps organizations identify assets of critical importance to their organiza- 


\section{An Enterprise Security Program and Architecture to Support Business Drivers}

\section{Brian Ritchot}

tion. Attempting to counter emerging cyberthreats without a clear understanding of the business needs of an organization will result in ineffective security controls and practices. When security is considered in the context of the enterprise, as both an enabler and means of assuring business success, control improvements can be tailored to the environment to address more sophisticated and complex threat scenarios. In the cases of 10 million new malwares every six months, intellectual property theft in the billions of dollars, and sophisticated intrusions such as Stuxnet, organizations are often incapable of prioritizing security initiatives, and they default to technical solutions without proper identification of critical assets and information. The cyber-challenge requires that organizations be better equipped than the threats acting against them, and an enterprise security architecture provides this capability.

\section{Conclusion}

For information assurance to effectively strike the appropriate balance between the protection of information and making the correct information easily assessable to authorized parties, an enterprise security architectures needs to be developed with a focus on business goals. An enterprise security architecture should provide a means of mitigating risk while also supporting the business to pursue new opportunities.

The approach described in this article provides security practitioners and senior executives with the knowledge and foundational information needed to connect security performance to business performance. As the IT threats facing an organization continue to grow in volume and variety, a reasonable approach is needed to address threats while continuing to support the operations of the business. Organizations that focus solely on the eradication of threats and vulnerabilities when developing security architecture risk creating an environment where security becomes an obstacle to operations. A business-driven approach to security architecture helps organizations prioritize where controls are needed to protect critical information, and it helps them define what level of risk is acceptable.

\section{About the Author}

Brian Ritchot is a Senior Information Security Consultant with Seccuris Inc, specializing in the implementation and delivery of intrusion-detection solutions, vulnerability assessment, network analysis, and security architecture. With 11 years of prior experience in the federal government, Brian has developed skills and expertise to support the detection, discovery, and mitigation of cyberthreat activity. Brian has led and managed several high-profile teams and projects to deliver operational security solutions that monitor and protect systems of importance to the Government of Canada. Brian now focuses his time in the private sector, helping a variety of customers across the critical infrastructure sector with their IT security needs. These activities span enterprise security architecture development, incident response and handling, vulnerability assessments, forensic investigations, and specialized IT security expertise to mitigate sophisticated cyberintrusions.

Citation: Ritchot, B. 2013. An Enterprise Security Program and Architecture to Support Business Drivers. Technology Innovation Management Review. August 2013: 25-33.

Keywords: security architecture, risk, cybersecurity, information assurance, cyberthreats, information risk, information security 